\title{
The Systemic Effects of Blood Flow Restriction Training: A Systematic Review
}

\author{
Bradley C Miller, PT, DPT, CSCS $^{1}$, , Alexander W Tirko, PT, DPT ${ }^{1}$, Justin M Shipe, PT, DPT', Olivia R Sumeriski, PT, DPT', \\ Kelley Moran, PT, EdD, DPT, ATC, CSCS ${ }^{1}$ \\ 1 Department of Physical Therapy, Misericordia University \\ Keywords: psychosocial, endocrine, cardiovascular, musculoskeletal, systemic effects, blood flow restriction \\ https://doi.org/10.26603/001c.25791
}

\section{International Journal of Sports Physical Therapy}

Vol. 16, Issue 4, 2021

\section{Background}

Blood flow restriction (BFR) training has been reported to have significant benefits on local skeletal muscle including increasing local muscle mass, strength, and endurance while exercising with lower resistance. As a result, patients unable to perform traditional resistance training may benefit from this technique. However, it is unclear what effects BFR may have on other body systems, such as the cardiovascular and pulmonary systems. It is important to explore the systemic effects of BFR training to ensure it is safe for use in physical therapy.

\section{Purpose}

The purpose of this study was to systematically review the systemic effects of blood flow restriction training when combined with exercise intervention.

\section{Study Design}

Systematic review.

\section{Methods}

Three literature searches were performed: June 2019, September 2019, and January 2020; using MedLine, ScienceDirect, PubMed, Cochrane Reviews and CINAHL Complete. Inclusion criteria included: at least one outcome measure addressing a cardiovascular, endocrinological, systemic or proximal musculoskeletal, or psychosocial outcome, use of clinically available blood flow restriction equipment, use of either resistance or aerobic training in combination with BFR, and use of quantitative measures. Exclusion criteria for articles included only measuring local or distal musculoskeletal changes due to BFR training, examining only passive BFR or ischemic preconditioning, articles not originating from a scholarly peer-reviewed journal, CEBM level of evidence less than two, or PEDro score less than four. Articles included in this review were analyzed with the CEBM levels of evidence hierarchy and PEDro scale.

\section{Results}

Thirty-five articles were included in the review. PEDro scores ranged between 4 and 8, and had CEBM levels of evidence of 1 and 2. Common systems studied included cardiovascular, musculoskeletal, endocrine, and psychosocial. This review found positive or neutral effects of blood flow restriction training on cardiovascular, endocrinological, musculoskeletal, and psychosocial outcomes.

\footnotetext{
Corresponding author: 


\section{Conclusions}

Although BFR prescription parameters and exercise interventions varied, the majority of included articles reported BFR training to produce favorable or non-detrimental effects to the cardiovascular, endocrine, and musculoskeletal systems. This review also found mixed effects on psychosocial outcomes when using BFR. Additionally, this review found no detrimental outcomes directly attributed to blood flow restriction training on the test subjects or outcomes tested. Thus, BFR training may be an effective intervention for patient populations that are unable to perform traditional exercise training with positive effects other than traditional distal muscle hypertrophy and strength and without significant drawbacks to the individual.

\section{Level of Evidence}

$1 \mathrm{~b}$

\section{INTRODUCTION}

Blood flow restriction (BFR) training has been found to have significant benefits for skeletal muscle development. BFR uses a belt or tourniquet applied to the proximal portion of an extremity to partially or fully occlude blood flow in order to stimulate muscular adaptations that improve muscular mass and strength. ${ }^{1}$ Traditionally, when attempting to improve muscle mass and strength, high intensity resistance training using loads of $\sim 70-85 \%$ of a one-repetition max (1-RM) is most often indicated. ${ }^{2}$ However, researchers have found that BFR in conjunction with low load resistance training, cardiovascular endurance training, and other forms of exercise not generally indicated to improve muscular mass/strength also cause these muscular adaptations. ${ }^{2,3}$ These findings may make the use of BFR valuable in the rehabilitation of patients who may not be able to perform high load resistance training such as the elderly, patients undergoing rehabilitation, recovering athletes, or in patients with other medical conditions such as renal disease, metabolic dysfunction, heart disease, or medically compromised high risk patients. Findings also suggest that those using BFR during training such as a cycling exercise program may receive the benefits of increased skeletal muscle mass and strength along with improved cardiovascular/ muscular endurance. ${ }^{4}$

Typically, research on BFR training reports the localized changes in muscle mass, strength, and muscle endurance of the extremity on which the cuff is applied. Research on BFR training has suggested other possible benefits or detriments involving systems other than the musculoskeletal system; however, these effects have not been determined conclusively. The focus of BFR is to cause beneficial adaptations to local skeletal muscle, but there is limited information reported on the effects of BFR training on other body systems. Multiple systems may be affected by BFR training, but a thorough analysis of these effects is still needed.

In order to better understand BFR and its overall impact on the human body, the purpose of this study was to systematically review the systemic effects of blood flow restriction training when combined with an exercise intervention. By further understanding the systemic effects of BFR training, clinicians may be able to incorporate this technique safely in the rehabilitation of patients who cannot perform high load resistance or aerobic training.

\section{METHODS}

An original database search was completed in June 2019 with a focus on the topic of the systemic effects of blood flow restriction (BFR) training. The following databases were used in the search: MedLine, ScienceDirect, PubMed, Cochrane Reviews and CINAHL Complete. Initial search terms included "blood flow restriction", “occlusion training”, "restriction of blood flow", "systemic effects", "blood flow restriction training”, "partial occlusion”, "effect or effects". Search criteria were filtered by article type (research articles, practice guidelines) and the year range 2009-2020. Titles and abstracts of articles were assessed by one of the four authors and a hand search of the systematic reviews by four authors yielded additional articles that were deemed relevant based on their titles and abstracts. Exclusion criteria for articles included research focusing on local musculoskeletal changes due to BFR training, studies examining passive BFR or ischemic preconditioning, and articles that did not originate from a scholarly peer-reviewed journal. Studies with CEBM level of evidence less than two were excluded, as the focus of this article was to review metaanalyses, RCTs, and cohort studies. Additionally, studies with PEDro scores less than 4 were excluded, as this score has been used previously to delineate "poor" quality from "fair", "good", and "excellent" quality studies.5,6 Four researchers calculated PEDro scores and CEBM levels of evidence and came to mutual agreement regarding when articles should be excluded due to inadequate quality. Articles were included if researchers used clinically available blood flow restriction equipment, used resistance or aerobic training in combination with BFR, used quantitative outcome measures, and were not dismissed by the exclusion criteria.

Updated database searches were completed in September 2019 and January 2020. These articles were screened using their titles and abstracts for relevance, inclusion, and exclusion criteria, as well as Oxford Centre of Evidence-Based Medicine level of evidence by one of four researchers. If deemed appropriate, the author evaluated the entire text for quality using the PEDro scale. If the article was a systematic review a hand search of references was performed, and results were assessed.

After the June 2019, September 2019, and January 2020 searches were completed, Inter-library loans through Misericordia University and ResearchGate requests were completed for articles that were unable to be accessed through 
the original databases. Any articles not received or granted access by February 1, 2020 were not included in the review due to a lack of access.

\section{RESULTS}

The initial search completed in June 2019 produced 2299 results. After refining parameters and removing duplicates, 281 articles were considered appropriate. Upon assessment of the abstracts and titles of all 281 articles, 30 articles and three systematic reviews were deemed appropriate for use in this review. After hand searching the systematic reviews, 41 additional articles were found and determined to be suitable. The updated search in September 2019 yielded 86 results, of which five articles were deemed appropriate. The updated search in January 2020 yielded 98 results, and six articles and one systematic review were determined to be suitable. Upon hand search of the systematic review, eight additional articles were located and considered to be appropriate. Ninety total articles were reviewed, and after 55 were excluded due to either lack of relevance, quality, or access, a total of 35 articles were included in the systematic review. Appendix 1 summarizes the characteristics and results of studies included in this review. Figure 1 describes the search timeline and methodology.

\section{CARDIOPULMONARY}

A paramount concern regarding the application of BFR training is the effects partial vascular occlusion has on cardiovascular and pulmonary health. The search found articles investigating the effects on maximal oxygen consumption (VO2 Max), 4,7-11 vascular stiffness and compliance, ${ }^{3,12-19}$ systolic and diastolic blood pressure (SBP and DBP, respectively) responses, ${ }^{3,16-23}$ heart rate (HR), ${ }^{7,16-19,24}$ stroke volume (SV), ${ }^{17,18}$ cardiac output (CO), ${ }^{17,18}$ ankle brachial pressure index (ABI), 3,19 and functional endurance in healthy adults ${ }^{9}$ and those with renal disease $^{25}$ and heart failure. ${ }^{26}$

\section{BLOOD PRESSURE}

Five studies examined the effects of BFR on systolic and diastolic blood pressure responses. ${ }^{3,16-23}$ In young adults using BFR during resistance training for hypertrophy it appears the modality causes no differences in SBP or DBP responses both during and after exercise when using between $50-80 \%$ aortic occlusion pressure at the proximal thighs or proximal upper arms and less than 20\% 1-RM. 3,22 In a group of young men performing six week bench press training with either proximal arm BFR at pressures of 160 mmHg and 30\% 1-RM (BFR group) or no occlusion and 75\% 1-RM (HIT group), Ozaki and colleagues found thigh SBP increased significantly in the HIT group compared to the BFR group, while both groups' resting SBP and DBP remained unchanged throughout the intervention period. ${ }^{16}$

In young adults performing BFR and aerobic exercise with occlusion pressures between 40-60 mmHg and 50\% maximum heart rate (HR Max), SBP and DBP responses were found to be similar to those traditionally found with aerobic intensities of $62-85 \%$ HR max. With appropriate in- dividualized dosing, aerobic exercise with BFR could provide an appropriate stimulus for aerobic adaptation. Two studies using a 15-minute treadmill test with bilateral lower extremity $160 \mathrm{mmHg}$ occlusion found central SBP and DBP responses were significantly greater than those seen in nonoccluded treadmill testing. ${ }^{17,18}$

In older adults one study found that occlusion pressures of $196 \mathrm{mmHg}(+/-18 \mathrm{mmHg})$ resulted in no differences in SBP and DBP responses between two cohorts of older adults performing upper extremity exercises with or without BFR. ${ }^{19}$

\section{HEART RATE, STROKE VOLUME, AND CARDIAC OUTPUT}

Six articles examined the effects of BFR on heart rate responses or cardiac output.7,8,16,18,19,24 The majority of studies evaluating heart rate (HR) found higher HR responses compared to intensity matched controls with both strengthening and aerobic based exercise.7,16-18,24 One study by Yasuda, Fukumura, and Yusuke reported no significant differences in the HR responses of two groups of older adults performing elastic band resistance training with or without BFR, when compared to pre-intervention. ${ }^{19}$ Two studies found smaller increases in stroke volume (SV) while performing aerobic treadmill sessions for BFR groups using $160 \mathrm{mmHg}$ bilateral lower extremity occlusion compared to controls. These studies also evaluated cardiac output (CO) and found both groups increased CO similarly in response to treadmill exercise. ${ }^{17,18}$

\section{VASCULAR STIFFNESS AND COMPLIANCE}

Nine articles evaluated the effects of BFR on vascular compliance or endothelial function. ${ }^{3,12-19}$ Ozaki et al. reported arterial stiffness increased more prominently in BFR compared to control when using $50-80 \%$ AOP. ${ }^{16}$ Other studies found no difference in arterial responses to exercise when comparing BFR to controls via brachial artery diameter, ankle-brachial index, flow-mediated dilation, and cardio-ankle vascular index. ${ }^{3,19}$ Ozaki et al. in 2013 found carotid arterial compliance was maintained in their BFR cohort using $160 \mathrm{mmHg}$ bilateral proximal upper arm occlusion during bench press training, compared to a $21 \%$ decrease in carotid compliance in controls. ${ }^{16}$ Ozaki et al. in 2011 found occlusion pressures up to $200 \mathrm{mmHg}$ applied during walk training to bilateral proximal thighs resulted in similar increases in carotid artery compliance compared to traditional walk training. ${ }^{13}$ Shimizu et al. reported BFR using femoral SBP increased transcutaneous oxygen pressure of the foot compared to both control and pre-intervention levels. ${ }^{14}$

\section{$\mathrm{VO}_{2}$ PEAK AND $\mathrm{VO}_{2} \mathrm{MAX}$}

Six articles in this review assessed the effects of BFR on $\mathrm{VO}_{2}$ peak and/or $\mathrm{VO}_{2}$ max. ${ }^{4,7-11}$ Two studies evaluating $\mathrm{VO}_{2}$ peak found concurrent resistance training and aerobic exercise or treadmill exercise resulted in similar increases or no change when using occlusion pressures of $50 \%$ AOP or $110-200 \mathrm{mmHg}$, respectively. ${ }^{8,9}$ Three studies evaluating the impact of BFR with aerobic exercise on $\mathrm{VO}_{2}$ max and found mixed results. Held and colleagues found an average 


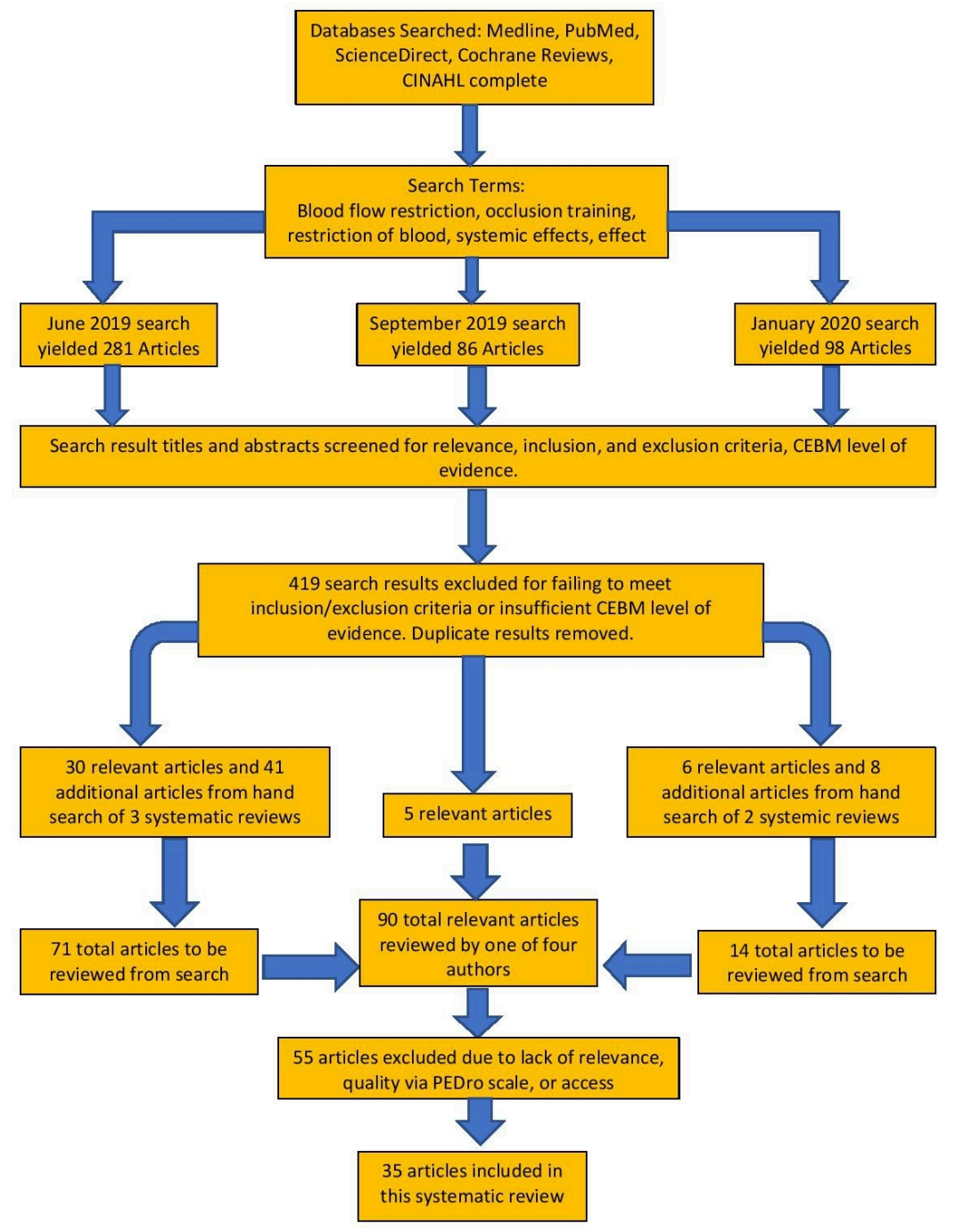

Figure 1. Search timeline and methodology flowchart

improvement of 9.6\% in $\mathrm{VO}_{2}$ max of elite rowers when using elastic wrapped BFR during low intensity row training, significantly more than exercising controls. ${ }^{10}$ Oliveira et al. used $18 \mathrm{~cm}$ wide cuffs at $140-200 \mathrm{mmHg}$ pressure and found similar increases in $\mathrm{VO}_{2}$ max with $29.4 \%$ of the high intensity training group volume. ${ }^{4}$ Paton, Addis, and Taylor found similar increases in $\mathrm{VO}_{2}$ max between BFR and control with the same exercise intensity (running speed as a percentage of peak running velocity). ${ }^{7}$ Mendonca et al. evaluated excess post-exercise oxygen consumption (EPOC) using 200 $\mathrm{mmHg}$ occlusion pressure with a $6 \mathrm{~cm}$ wide cuff and found walking with BFR increased EPOC post-intervention significantly more than walking without the modality. ${ }^{11}$

\section{EXERCISE CAPACITY}

Three articles evaluated exercise capacity: one of healthy adults, ${ }^{9}$ one in patients with end stage renal disease (ESRD), ${ }^{25}$ and one in patients with heart failure (HF). ${ }^{26}$

In healthy older adults, it appears BFR in combination with six weeks of treadmill walking can improve functional ability as evaluated by Timed-Up-and-Go and 30 Second Sit-to-Stand scores significantly more than walking alone. ${ }^{9}$
In two of the most clinically relevant cardiovascular studies, BFR was found to improve 6-minute walk test distances $17 \%$ (compared to $1.5 \%$ improvement in exercising control) in patients with ESRD on hemodialysis. ${ }^{25}$ These patients used cycle ergometry and $50 \%$ AOP while receiving dialysis treatment. The exercise was performed for 20 minutes of the four-hour dialysis session three times per week, and the researchers reported no adverse effects correlated to the use of the modality. In patients with post-infarction HF with an average ejection fraction of $52.9 \%$, BFR at an average $208 \mathrm{mmHg}$ to bilateral proximal thighs with cycle ergometry was found to significantly improve $\mathrm{VO}_{2} / \mathrm{W}$ and anaerobic threshold compared to exercise matched controls. ${ }^{26}$

\section{SYSTEMIC MUSCULOSKELETAL}

Along with a large array of systemic cardiopulmonary effects, BFR training has also demonstrated a variety of systemic effects on the musculoskeletal system. ${ }^{16,27-32}$ Previous research on the technique has focused on localized muscle hypertrophy, strength, and endurance following application and methodization of BFR training. ${ }^{27,28} \mathrm{~A}$ vast majority of previous research compares the results of low- 
intensity BFR training to high intensity resistance training without BFR in order to determine if low-intensity BFR may be a comparable training stimulus. Although not all the articles were conclusive of definite systemic musculoskeletal effects, many of the studies did report systemic effects involving the musculoskeletal system that were attributed to BFR. When compared to various other training methods or techniques, many of the findings demonstrated noteworthy, or at least comparable, results to other groups. Interestingly, six of the seven articles included in the musculoskeletal portion of the review reported distal or contralateral strengthening, ${ }^{16,27-31}$ hypertrophy, ${ }^{16,27,29-32}$ or muscle function adaptation $16,27-32$ that can be attributed to systemic effects of BFR training.

\section{ENDOCRINE}

\section{MUSCULAR ADAPTATION}

Cook found that when comparing two groups of young men performing identical exercise programs, one group using BFR and a control group, participants training with BFR see a significantly greater increase in free testosterone concentrations compared to the control. ${ }^{28}$

Another important factor in developing muscle, serum growth hormone $(\mathrm{GH})$ concentration was also found to have increased significantly more in elderly participants using BFR training techniques as compared to a non-BFR control group when performing identical low intensity resistance programs. ${ }^{14}$

Laurentino discovered BFR may induce muscular adaptations by inhibiting factors that are detrimental to muscle growth. ${ }^{33}$ Following 8 weeks of low intensity resistance training with BFR, myostatin (MSTN) mRNA gene expression was found to have significantly decreased (45\% decrease in BFR low intensity groups, $41 \%$ in non-BFR high intensity group), while Growth and Differentiation- Associated Serum Protein-1 (GASP-1) and MAD-related protein (SMAD-7) gene expressions significantly increased (GASP-1: 82\% increase in BFR groups compared to $79 \%$ in non-BFR; SMAD-7: 88\% increase in BFR group compared to $66 \%$ in non-BFR group). ${ }^{33}$

In a research study using a sample of healthy elderly men, Karabulut reported no significant change in interleukin 6 (IL-6), insulin-like growth factor-1 (IGF-1), and free testosterone between participants in high-intensity resistance training, low intensity resistance training with $\mathrm{BFR}$, and control groups. ${ }^{34}$

\section{OSTEOBLASTIC ACTIVITY}

Karabulut et al.'s study investigated BFR's influence on bone alkaline phosphate (ALP) and C-telopeptide of Type-1 collagen (CTX) as well as ALP/CTX ratio. ${ }^{35}$ In 6 weeks, participants performing low intensity resistance training with $\mathrm{BFR} /$ vascular restriction (LI-VRT) and high-intensity resistance training only (HI-RT) demonstrated significant increases in ALP concentration and improved bone ALP/CTX ratio as compared to a control group. ${ }^{35}$ LI-VRT and HI-RT saw $21 \%$ and $23 \%$ increases in concentrations, respectively, while the control had an $4.7 \%$ increase. LI-VRT and HI-RT saw decreases in CTX concentrations of $7.7 \%$ and $4.1 \%$, respectively, while the control group had a $3.3 \%$ increase in CTX concentration.

\section{METABOLIC STRESS}

Multiple studies have found that when using BFR in conjunction with low resistance exercise, blood lactate levels are significantly higher than control groups and levels are similar to that produced by high intensity training. 4,36 Oliveira et al., found significant increases in blood lactate accumulation (measured before and after treatment) between participates performing low intensity exercises with BFR $(16 \%+/-13 \%)$ and those performing low intensity exercise without BFR $(6 \%+/-4 \%) .{ }^{4}$ Neto found that groups participating in low intensity exercises with BFR (5.0\% increase) provide similar levels of blood lactate accumulation as compared to high intensity exercises without BFR (5.2\% increase). ${ }^{36}$ Shimizu et al. found when comparing BFR groups to non-BFR, participants using BFR have significant increases in lactate levels (non-BFR: $10.3+/-5.3$ before, $34.3+/-13.3$ after; BFR: $8.2+/-3.6$ before, $49.2+/-16.1$ after, $\mathrm{mg} \mathrm{dL}^{-1}$ ) as well as increases norepinephrine (non-BFR: 472.4 +/- 136.8 before, 662.1 +/- 201.5 after; $619.5+/-243.7$, $960.2+/-373.7$ after, $\mathrm{mg} \mathrm{dL}^{-1}$ ).

Okita et al research finds that metabolic stress is induced by decreases in phosphocreatine and intramuscular $\mathrm{pH}$. Participants performing low intensity exercises do not have significant decrease in intramuscular $\mathrm{pH}$ while participants using intermittent BFR (-.10 pH) and continuous BFR (-.125 $\mathrm{pH}$ ) have significant decrease in $\mathrm{pH}$.

\section{HIGH RISK PATIENTS}

Tanaka and Takarade's research investigated the effects of BFR with a patient population of 30 men (mean age of 60.7 +/-11 years) with a history of CHF. Results after six months of exercise training showed no change in the serum triglyceride, high-density lipoprotein, LDL-C, total cholesterol, glucose, and HbA1c levels. ${ }^{26}$ Additionally, after the sixmonth time frame, brain natriuretic peptide levels decreased significantly. ${ }^{26}$

\section{PSYCHOSOCIAL FACTORS}

The effects of BFR are not only that of a physical nature, but that of a patient's psychological state. Furthermore, not only may the effects of BFR impact a patient's psychological state, but may also affect a patient's physical state/performance.

To assess these effects multiple studies have assessed an individual's rate of perceived exertion (RPE), ${ }^{19,36,37}$ overall mood, ${ }^{38,39}$ pain levels, ${ }^{37}$ and overall levels of discomfort. ${ }^{40}$

When investigating effects on RPE, studies have found when initially training with BFR and low load resistance training RPE was increased in most individuals as compared to traditional high load resistance exercise. ${ }^{19,36,37}$ While RPE initially did increase in most cases during the beginning stages of BFR, RPE rates decreased over a longer period of time lasting 8 weeks when compared to the traditional high load resistance training. ${ }^{40}$ When comparing the effects 
of RPE between continuous vs intermittent BFR, the average RPE levels were lower when using intermittent BFR as compared to using continuous BFR. 36

To evaluate general pain level and level of discomfort, a 0-10 general pain scale and a BORG discomfort scale was used to track these complaints over the BFR application period. In a study using bilateral leg press, both high and low load resistance training using 80\% and 30\% 1 RM showed higher RPE and pain ratings after exercise to muscular failure than a BFR group training with 30\% 1 RM and using 4 sets of 15 repetitions scheme. ${ }^{37}$ In a separate study comparing groups performing upper extremity exercises, those participants performing the same exercises with BFR reported significant increases in discomfort rating. ${ }^{40}$

The utilization of BFR with resistance training has been found to have a significant effect on an individuals' overall mood state. Silva 2018 measured mood state, total mood disturbance, and RPE before and after exercising with and without BFR. ${ }^{38}$ These researchers found that BFR induced an acute negative effect on mood state, total mood disturbance, and increased overall participant fatigue. ${ }^{38}$ Silva 2019 research investigated mood state after aerobic exercise with BFR and found BFR to cause acute impairments in mood state and RPE in most individuals. ${ }^{39}$ However, this affect was comparable to the effects found with traditional high load resistance training. ${ }^{39}$

\section{DISCUSSION}

\section{CARDIOPULMONARY}

\section{BLOOD PRESSURE}

Previous studies have shown a correlation between resistance training and training-induced arterial stiffening, and a low level of arterial compliance has been shown to contribute to heart disease and impaired baroreflex sensitivity. ${ }^{20,21,23}$ The studies included in this review suggest BFR using occlusion pressures less than approximately 200 $\mathrm{mmHg}$ does not lead to detrimental blood pressure responses in healthy adults. Rather, BFR under these parameters causes similar blood pressure responses as traditional exercise, when prescribed appropriately.

\section{HEART RATE, STROKE VOLUME, AND CARDIAC OUTPUT}

When evaluated together, these studies show the application of BFR does not change CO but does decrease relative SV amount and increase HR response accordingly to maintain appropriate CO. These findings suggest the increased pressure from the occlusion cuffs, when using pressures of less than $160 \mathrm{mmHg}$ or $7 / 10$ subject perceived pressure with resistance or aerobic exercise, does not negatively impact cardiac output. Additionally, the exaggerated HR response may be beneficial for improving cardiac conditioning in those who cannot handle traditional stimuli needed to attain $70-85 \%$ HR Max. ${ }^{41}$

\section{VASCULAR STIFFNESS AND COMPLIANCE}

When using BFR with aerobic exercise, Renzi et al. found increased arterial stiffness (measured using SV/PP) and de- creased flow-mediated dilation when using $160 \mathrm{mmHg}$ with walk training. ${ }^{18}$ Iida, Nakjima, and Abe found a six week walking program with $140-200 \mathrm{mmHg}$ bilateral lower extremity BFR significantly increased maximal venous outflow and venous compliance compared to no change in their control. ${ }^{15}$ Ozaki et al. in the year 2011 found walk training with 140-200 mmHg occlusion pressure did not lead to significantly different changes in carotid artery compliance compared to control. ${ }^{13}$ This finding is significant because these authors also found a significant increase in thigh muscle cross sectional area, knee flexion torque, and knee extension torque in the BFR group compared to control. While this finding is outside the scope of this literature review, the possibility of improving muscle hypertrophy and carotid artery compliance concurrently using BFR with aerobic training is worth future investigation. ${ }^{13}$

BFR effects on vascular compliance and stiffness varied among studies. ${ }^{13,14,16-18}$ However, it is important to identify the trend of these results: higher relative pressures tend to decrease the benefit and may incur unfavorable changes to the vascular system. A possible explanation of the negative outcomes seen with vascular changes may be explained by the pressure gradient created by narrow width occlusion cuffs. Studies of surgical tourniquets have found an inverse relationship between the width of occlusion cuffs and the pressure required to attain total limb occlusion with a cuff width to limb circumference ratio less than 0.5 requiring sub-systolic pressures. ${ }^{42,43}$ In BFR training a similar concept may be paramount to elicit favorable adaptations using the lowest pressures and lowest pressure gradients possible.

\section{$\mathrm{VO}_{2}$ PEAK AND $\mathrm{VO}_{2} \mathrm{MAX}$}

These studies show while benefits to $\mathrm{VO}_{2}$ peak may not be attainable, $\mathrm{VO}_{2}$ max can be significantly improved given an appropriate occlusion pressure and training stimulus. Importantly it appears $\mathrm{VO}_{2}$ max can be improved to a similar degree as traditional aerobic exercise, and this benefit can be realized with significantly less volume when augmented with BFR. Additionally, because BFR seems to increase the relative intensity and cumulative oxygen deficit of an aerobic activity, lower stimuli may be sufficient to incur benefits to $\mathrm{VO}_{2}$ max. ${ }^{11}$ This may be especially valuable to those who are unable to exercise at intensities high enough to improve or maintain aerobic capacity.

\section{EXERCISE CAPACITY}

These studies are novel in their use of medically complex patients and display how appropriately dosed BFR training with aerobic exercise can safely and significantly improve functional capacity.

In healthy older adults Abe and colleagues found no improvement in aerobic capacity following six weeks of treadmill walking with BFR, however the BFR group did significantly improve chair stand and Timed-Up-and-Go performance compared to an active control. Importantly, the authors did not reach the 50\% HR Max reserve intensity they hypothesized was required to elicit aerobic changes, and previous research included in their study concluded "similarly intense walk training without BFR elicits little or 
no effect on aerobic capacity". 9

Cardoso and colleagues noted the improvement realized in their study could be due in part to the higher metabolic stress generated by hypoxia from BFR, a hypothesis that has been put forth to explain strength and hypertrophy gains experienced with this modality.

When using BFR with post-infarction heart failure patients, Tanaka and others found significant improvements in anaerobic threshold using BFR with cycle ergometry. This study is significant in its use of high occlusion pressures (208.7+/- $7.4 \mathrm{mmHg}$ ) with exercise in medically complex patients with no adverse effects of exercise training noted. ${ }^{26}$ While a small study, the significant improvement in anaerobic threshold compared to exercising control without adverse training effects is encouraging for the safe use of this intervention in this population and others with cardiovascular conditions.

\section{SYSTEMIC MUSCULOSKELETAL}

\section{STRENGTH}

Several studies have determined that low-load BFR training may increase strength contralateral and distal to cuff or tourniquet placement. ${ }^{16,27-31}$ Compared to high-load resistance training, low-load with BFR has shown to produce similar, and sometimes more significant, effects on muscle strength. ${ }^{27}$ Cook et al. discovered a systemic effect of increased muscle strength of the upper extremities when occluding bilateral lower extremity blood flow. ${ }^{28}$ BFR training with similar exercise regimen as the control group demonstrated a significantly greater increase in bench press strength compared to the non-BFR group, $1.4 \% \pm 0.8 \% .28$ Occlusion of lower extremities increasing upper-body strength more than a group without BFR validates the idea that there is a systemic effects on the body, but the overall mechanism of these effects are yet to be fully understood. ${ }^{28}$

May et al. used lower extremity BFR training following unilateral arm exercises and during lower extremity exercises to demonstrate a larger increase in trained arm strength of the BFR group compared to the trained arm of the control group, which displays a possible systemic effect stemming from partial blood flow occlusion. The systemic or "transfer effect" on upper body strength is supported by the increased elbow flexion 1-RM in the untrained arm of the experimental group. ${ }^{29}$ May et al. ${ }^{29}$ and Cook et al. ${ }^{28}$ hypothesized that noted systemic musculoskeletal effects of BFR can be attributed to what has been called the "cross-transfer phenomenon". According to May et al., cross-transfer is common with unilateral resistance training, with contralateral sites. However, there has not been much research on isolated sites and their effect on other sites; it is believed in this study BFR of the lower extremities had a cross-transfer effect on the upper extremities. May et al. also suggests that because cross-sectional area of musculature grew similarly between extremities, the strength transfer was not due to hypertrophy of muscle, but rather a neuromuscular adaptation. ${ }^{29}$

Bowman et al. also found contralateral lower extremity strength gains in the low-load BFR group compared to lowload training alone. ${ }^{27}$ The control group performed low- load resistance training of the lower extremities, whereas the BFR group performed the same exercises but with partial occlusion to blood flow of the upper thigh of one lower extremity. ${ }^{27}$ Bowman hypothesizes that improved strength and increases in muscle hypertrophy are due to metabolic stress triggering consequent metabolic, adrenergic, and hormonal changes that eventually lead to muscular adaptation. ${ }^{27}$ Both Ozaki et al. ${ }^{16}$ and Thiebaud et al. ${ }^{30}$ discovered similar effects on strength gains between BFR and non-BFR groups. Ozaki et al. found that when occluding upper extremity blood flow, the BFR group produced similar gains in bench press 1-RM compared to the non-BFR group, but the BFR group was working at $45 \%$ lower intensity. ${ }^{16}$ Thiebaud et al. found that BFR with cuffs placed on upper extremities demonstrated significant increases in strength of chest press, shoulder press, and seated row equal to the non-BFR group with no significant differences between the two. ${ }^{30}$ Again, the BFR group was exercising at a lower intensity, but still demonstrated equal gains in strength.

Yasuda et al. found that when the training intensities remain the same, and the only difference between two groups is BFR of the upper extremities, the BFR group demonstrated more significant changes in bench press 1-RM compared to the non-BFR group. Because they also found increases in muscle hypertrophy, Yasuda et al. believes strength gains cannot be due to neural adaptation alone. ${ }^{31}$

\section{HYPERTROPHY}

Both Thiebaud et al. ${ }^{30}$ and Ozaki et al. ${ }^{16}$ compared moderate to high intensity resistance training to low intensity resistance training with BFR and their effect on, cross-sectional area (CSA) of pectoralis major when occluding blood of the upper extremities. Findings of the studies determined that even with a lower workload, the BFR group experienced almost equivalent pectoralis muscle hypertrophy as their counterpart. ${ }^{16,30}$ Thiebaud et al. also found that there were no significant differences between groups concerning lower extremity muscle hypertrophy, but both groups experienced significant changes in upper thigh muscle thickness with the BFR group exercising at a lower intensity. ${ }^{30}$ With unilateral LE occlusion, Bowman et al. found greater increase in contralateral BFR lower extremity compared to non BFR group. ${ }^{27}$ May et al. found that, with similar exercise regimens, BFR applied to the most proximal portion of bilateral lower extremities did not promote a significantly different change in upper extremity muscle hypertrophy compared to the non-BFR group. ${ }^{29}$ Because there was strength gain without an increase in muscle size, it is clear why May attributes the systemic increase in strength attained from BFR to neuromuscular adaptation and not to musculature hypertrophy. ${ }^{29}$ This finding is in contrast to previous findings from Yasuda et al. who reported a measurable increase in muscle size. Yasuda et al. determined that, with identical resistance training protocol, bilateral upper extremity BFR promotes significant increase in unrestricted chest muscle hypertrophy (pectoralis major) compared to no BFR. ${ }^{31} \mathrm{Un}$ like multiple previous studies, Sakamaki et al. compared a BFR exercise group to a non-BFR exercise group and determined no or minimal difference in systemic muscle hypertrophy of gluteus maximus and iliopsoas muscles between 
the groups. ${ }^{32}$ The BFR cuffs were placed around the most proximal portion of each leg, and the regimen consisted of three weeks of treadmill walking training. ${ }^{32}$ Their findings may be due to the fact that the training regimen was not as intense as resistance training exercises, making it less likely muscles would hypertrophy.

\section{ENDOCRINE}

Multiple studies have demonstrated that BFR has a profound effect on the endocrine system by affecting the hormones, blood factors, and biological complexes which control the human body. 4,14,28,33-36,44 These changes might not only impact muscle, but also multiple body systems. Knowing the endocrinological changes induced by BFR is not only important to understand how BFR is an effective tool, but if/how it can be a danger to potential patients.

\section{MUSCULAR ADAPTATION}

BFR's greatest potential as a therapeutic tool comes from its ability to improve muscle strength and induce muscle hypertrophy without placing the physical stress of high intensity resistance training on the body. These muscular adaptations may come as a result of the hormonal changes BFR induces.

Increases in free testosterone as well as serum growth hormone may be one of the mechanisms in which BFR helps induce muscular adaptation. ${ }^{14,28}$ Increases in these hormones promote the growth of muscle tissue allowing for potentially greater increases in strength as compared to changes induced by low intensity exercise only.

Laurentino et al. suggest that BFR promotes increased muscle growth through its effects on Myostatin (MSTN), Growth and Differentiation- Associated Serum Protein-1 (GASP-1) and MAD-related protein (SMAD-7) gene expressions. MSTN plays a role in controlling/inhibiting muscle growth, while GASP-1 and SMAD-7 play roles in inhibiting MSTN formation/function. As a result, the increases in GASP-1 and SMAD-7 expression will decrease the inhibitor actions of MSTN, and the decrease in MSTN expression means overall less active inhibition of muscle growth.

Karabulut's findings leave questions unanswered regarding the mechanism by which BFR may demand muscular adaptation. Finding that there was no significant change in growth factors/hormones, such as IL-6, IGF-1 and free testosterone, that would promote muscle growth may mean that it is not hormonal effects that cause muscular adaptation. 34

\section{OSTEOBLASTIC ACTIVITY}

An important benefit of resistance training, especially in the elderly, is its ability to increase bone density/mass. Increasing bone density and mass is important in order to prepare for degeneration with natural aging and prevents injury in the future. The results of Karabulut et al.'s research found that both treatment groups (low intensity resistance training with and without BFR) will benefit from a shift in bone turnover/metabolism that favors bone formation. However, this positive shift in osteoblastic activity shows that BFR in conjunction with low intensity exercise can facilitate improvements in bone density without placing the body through the physical stress of high intensity resistance training.

\section{METABOLIC STRESS}

One of the most significant endocrinological changes that is induced by BFR training is increased blood lactate levels. Studies have found that when using BFR in conjunction with low resistance exercise, blood lactate levels are significantly higher than control groups and levels are similar to that produced by high intensity training. ${ }^{4,36}$ Increased lactate levels lead to increased muscle soreness and fatigue, which might discourage participants from continuing the training practice/therapy service. However, it would seem that this increase in lactate levels is an important mechanism for beneficial muscular adaptations as one theory behind BFR's effectiveness suggests that the low oxygen environment promotes high metabolic stress (by high lactate levels). This increased metabolic stress causes the process of muscle damage and repair, which leads to muscle growth. Not only is it speculated that increases in lactate levels cause metabolic stress which induces muscular adaptation, but Okita finds that decreases in intramuscular $\mathrm{PCr}$ and intramuscular $\mathrm{pH}$ cause equivalent stress (with moderate resistance). Their studies suggest that these changes induce stress and drive muscular adaptation, similar to the effect of lactate.

Shimizu et al. conducted studies to identify the impact of BFR on endothelial function and peripheral circulation in the elderly while also looking at blood lactate levels (source of metabolic stress). The authors surmise that the increases in norepinephrine are needed in order to increase HR and BP to levels that allow sustainable, safe exercise while using BFR techniques, which induces significant metabolic stress. ${ }^{14}$ However, more research is needed to investigate if lactic acid and $\mathrm{pH}$ have the potential to reach levels in which the participant enters metabolic acidosis. Additionally, specific research is needed to evaluate risk of metabolic acidosis during BFR training in patients with diseases such as kidney failure. This need for further research should be expanded to investigate how the physical and metabolic stresses of BFR techniques affect a multitude of conditions.

\section{HIGH RISK PATIENTS}

To utilize BFR's potential, research needs to confirm that BFR may be used safely with patients that are medically compromised/high-risk. Tanaka and Takarade's research specifically investigated the effects of BFR on patients with CHF. Overall, their research finds that in a population of men (mean age of $60.7+/-11$ years) there were no noticeable adverse effects of BFR training in conjunction with aerobic exercise. ${ }^{26}$ Even though a goal of exercise is to improve cholesterol and glucose levels, a lack of change in these levels and no described adverse effects shows that the stress of BFR can be properly tolerated by elderly patients with CHF under proper guidance. ${ }^{26}$ However, there are significant limitations to this study as effects were not investigated for women of any age, men of younger age, and those 
with other significant diagnoses.

\section{PSYCHOSOCIAL FACTORS}

As discussed earlier, BFR does not only have physical effects but appears to have psychological effects as well. This psychological effect may deter an individual from participating in BFR training and may, furthermore, cause a negative impact to one's physical performance/state.

Increases in RPE may initially deter individuals as they are reporting feelings of having to exert more effort as compared to traditional training. ${ }^{19,36,37}$ This may be especially true in the field of physical therapy as we see many individuals that do not normally participate in strength training or may have individuals that are already poorly motivated to participate in physical activity. However, this review also shows RPE may initially be significantly higher, but with continued training, it appears most individuals build tolerance to the practice and RPE levels decrease when compared to traditional training. ${ }^{40}$ To potentially minimized the negative impact of elevated RPE, intermittent BFR may be more tolerable compared to continuous BFR as it is found to produce lower reports RPE over a training session. ${ }^{36}$

When looking at discomfort and pain ratings with BFR there is less definable changes. When comparing participants performing LE exercises, researchers suggest that those using BFR in conjunction with low intensity resistance training may have similar physical effects to those performing higher intensity exercise without BFR while participants using BFR complained of less pain over time. ${ }^{37}$ However, when measuring discomfort in participants performing upper UE exercise, BFR groups were found to have higher complaints of discomfort compared to their non-BFR counterparts. ${ }^{40}$ Further research will need to be conducted due to the different variables between these studies, but it appears that from this data, overall pain ratings decrease overtime with use of BFR while overall discomfort rating increased overtime.

Due to the negative effects to overall mood state and total mood disturbance, it is suggested that BFR is not to be used directly prior to athletic competitions. ${ }^{38}$ Decreased mood and the described participant fatigue caused by BFR may leave an athlete at a disadvantage compared to those that feel rested and prepared for competition.

While BFR has been found to have many positive physiological effects, is it important to consider the potential effects that it can have on the mood and psychological state. The benefits of a decreased RPE over time and negative effects on acute mood state need to be compared for each individual in order to determine whether this intervention is beneficial for them.

\section{LIMITATIONS}

While completing this research, several limitations were identified that could potentially affect the significance of the findings. While all studies were utilizing blood flow re- striction techniques in combination with exercise, the application and dosing parameters were widespread and not standardized between studies. It is unclear whether the results of each study would be significantly changed with different application and dosing parameters, which poses a limitation to this review. In addition to the non-standardization seen in dosing parameters, the studies utilized in this review did not provide diverse patient demographics. Most studies utilized a patient population between the age range of 18-39, offering little information on the older adult population. Similarly, the studies did not commonly include disease-specific populations, as most of the studies were performed on healthy individuals. This poses a limitation to the generalizability of this findings as they are limited to a mainly younger, healthy population. Finally, a potential conflict of interest is present due to several articles used in this review being authored or contributed to by Dr. Yoshiaki Sato, who is credited with inventing KAATSU training, a form of BFR, and holds several patents on BFR products. Studies coauthored by individuals related to this organization have been noted in Appendix 1. As these researchers may be invested in seeing beneficial impacts of this technique, their findings should be scrutinized.

\section{CONCLUSION}

The results of this systematic review suggest that blood flow restriction training has wide reaching effects on multiple body systems including cardiopulmonary, vascular, systemic musculoskeletal, and endocrine, as well as psychosocial factors. Overall, it does appear that BFR is beneficial to patients performing this style of training with currently no known adverse effects when dosed properly. In studies performed using patients with heart and renal disease, the use of BFR was not detrimental and even induced some benefits. It appears the greatest advantage of BFR is its ability to safely augment exercise intensity in both healthy and comorbid individuals. However, more research is needed before fully determining the long-term systemic effects of BFR. Further research is needed to investigate the appropriate dosing parameters, including ideal cuff width, pressure, and duration of partial occlusion. Once a "gold standard" BFR protocol is developed, the research of this review should be replicated to evaluate the reliability of the data.

\section{COI STATEMENT}

The authors of this systematic review report no conflicts of interest, financial or otherwise, in the production of this manuscript.

Submitted: December 22, 2020 CDT, Accepted: April 17, 2021 CDT 


\section{REFERENCES}

1. Iida H, Nakajima T, Kurano M, et al. Effects of walking with blood flow restriction on limb venous compliance in elderly subjects. Clin Physiol Funct Imaging. 2011;31(6):472-476. doi:10.1111/j.1475-097 $\underline{\mathrm{x} .2011 .01044 . \mathrm{x}}$

2. Slysz J, Stultz J, Burr JF. The efficacy of blood flow restricted exercise: a systematic review \& metaanalysis. J Sci Med Sport. 2016;19(8):669-675. doi:10.1 016/j.jsams.2015.09.005

3. Mouser JG, Mattocks KT, Dankel SJ, et al. Very-lowload resistance exercise in the upper body with and without blood flow restriction: cardiovascular outcomes. Appl Physiol Nutr Metab. 2018;44(3):288-292. doi:10.1139/apnm-2018-0325

4. de Oliveira MFM, Caputo F, Corvino RB, Denadai BS. Short-term low-intensity blood flow restricted interval training improves both aerobic fitness and muscle strength. Scand J Med Sci Sports.

2016;26(9):1017-1025. doi:10.1111/sms.12540

5. Oxford Centre for Evidence-Based Medicine: Levels of Evidence. University of Oxford. https://www.bcit.c a/files/library/pdf/bcit-ama_citation_guide.pdf. Published March 2009. Accessed March 22, 2021.

6. Foley NC, Teasell RW, Bhogal SK, Speechley MR. Stroke rehabilitation evidence-based review: Methodology. Top Stroke Rehabil. 2003;10(1):1-7. do i:10.1310/y6tg-1kq9-ledq-6418

7. Paton CD, Addis SM, Taylor LA. The effects of muscle blood flow restriction during running training on measures of aerobic capacity and run time to exhaustion. Eur J Appl Physiol. 2017;117(1):2579-2585. doi:10.1007/s00421-017-374 $\underline{5-3}$

8. Libardi CA, Chacon-Mikahil M, Cavaglieri C, et al. Effect of concurrent training with blood flow restriction in the elderly. Int J Sports Med. 2015;36(5):395-399. doi:10.1055/s-0034-1390496

9. Abe T, Sakamaki M, Fujita S, et al. Effects of lowintensity walking training with restricted leg blood flow on muscle strength and aerobic capacity in older adults. J Geriatr Phys Ther. 2010;33(1):33-40. doi:10.1 097/IPT.0b013e3181d07a73

10. Held S, Behringer M, Donath L. Low intensity rowing with blood flow restriction over 5 weeks increases VO2max during low intensity rowing: A randomized control trial. I Sci Med Sport.

2019;23(3):304-308. doi:10.1016/j.jsams.2019.10.002
11. Mendonca GV, Vaz JR, Pezarat-Correia P, Fernhall B. Effects of walking with blood flow restriction on excess post-exercise oxygen consumption. Int J Sports Med. 2015;36(3):e11-e18. doi:10.1055/s-0034-139550 $\underline{8}$

12. Fahs CA, Rossow LM, Thiebaud RS, et al. Vascular adaptations to low-load resistance training with and without blood flow restriction. Eur J Appl Physiol. 2013;114(4):715-724. doi:10.1007/s00421-013-2808-3

13. Ozaki H, Miyachi M, Nakajima T, Abe T. Effects of 10 weeks walk training with leg blood flow reduction on carotid arterial compliance and muscle size in the elderly adults. ANG. 2011;62(1):81-86. doi:10.1177/00 $\underline{03319710375942}$

14. Shimizu R, Hotta K, Yamamoto S, et al. Lowintensity resistance training with blood flow restriction improves vascular endothelial function and peripheral blood circulation in healthy elderly people. Eur J Appl Phys. 2016;116(4):749-757. doi:10.1 007/s00421-016-3328-8

15. Iida H, Nakajima T, Kurano M, et al. Effects of walking with blood flow restriction on limb venous compliance in elderly subjects. Clin Physiol Funct Imaging. 2011;31(6):472-476. doi:10.1111/j.1475-097 $\underline{\mathrm{x} .2011 .01044 . \mathrm{x}}$

16. Ozaki H, Yasuda T, Ogasawara R, SakamakiSunaga M, Naito H, Abe T. Effects of high-intensity and blood flow-restricted low-intensity resistance training on carotid arterial compliance: role of blood pressure during training sessions. Eur J Appl Physiol. 2013;113(1):167-174. doi:10.1007/s00421-012-2422-9

17. Sugawara J, Tomoto T, Tanaka H. Impact of leg blood flow restriction during walking on central arterial hemodynamics. Am J Physiol Regul Integr Comp Physiol. 2015;309(7):R732-R739. doi:10.1152/aj pregu.00095.2015

18. Renzi CP, Tanaka H, Sugawara J. Effects of leg blood flow restriction during walking on cardiovascular function. Med Sci Sports Exerc. 2010. $\underline{\mathrm{d}}$ oi:10.1249/MSS.0b013c3181bdb454

19. Yasuda T, Fukumura K, Uchida Y, et al. Effects of low-load, elastic band resistance training combined with blood flow restriction on muscle size and arterial stiffness in older adults. J Gerontol A Biol Sci Med Sci. 2015;70(8):950-958. doi:10.1093/gerona/glu084 
20. Miyachi M, Donato AJ, Yamamoto K, et al. Greater age-related reductions in central arterial compliance in resistance-trained men. Hypertension. 2003;41(1):130-135. doi:10.1161/01.hyp.000004764 9.62181.88

21. Miyachi M, Kawano H, Sugawara J, et al. Unfavorable effects of resistance training on central arterial compliance: a randomized intervention study. Circulation. 2004;110(18):2858-2863. doi:10.1161/01.C ir.0000146380.08401.99

22. Moriggi R, Di Mauro H, Dias S, et al. Similar hypotensive responses to resistance exercise with and without blood flow restriction. Biol Sport.

2015;32(4):289-294. doi:10.5604/230831862.1163691

23. Monahan KD, Tanaka H, Dinenno FA, Seals DR. Central arterial compliance is associated with ageand habitual exercise- related differences in cardiovagal baroreflex sensitivity. Circulation. 2001;104(14):1627-1632. doi:10.1161/hc3901.096670

24. Karabulut M, Garcia SD. Hemodynamic responses and energy expenditure during blood flow restriction exercise in obese population. Clin Physiol Funct Imaging. 2017;37:1-7. doi:10.1111/cpf.1225

25. Cardoso RK, Araujo AM, Del Vechio FB, et al. Intradialytic exercise with blood flow restriction is more effective than conventional exercise in improving walking endurance in hemodialysis patients: a randomized controlled trial. Clin Rehabil. 2020;34(1):91-98. doi:10.1177/0269215519880235

26. Tanaka Y, Takarada Y. The impact of aerobic exercise training with vascular occlusion in patients with chronic heart failure. ESC Heart Fail. 2018;5(4):586-591. doi:10.1002/ehf2.12285

27. Bowman EN, Elshaar R, Milligan H, et al. Proximal, distal, and contralateral effects of blood flow restriction training on the lower extremities: a randomized controlled trial. Sports Health. 2019;11(2):149-156. doi:10.1177/1941738118821929

28. Cook CJ, Kilduff LP, Beaven CM. Improving strength and power in trained athletes with 3 weeks of occlusion training. Int J Sports Physiol Perform. 2014;9(1):166-172. doi:10.1123/ijspp.2013-0018

29. May AK, Russell AP, Warmington SA. Lower body blood flow restriction training may induce remote muscle strength adaptations in an active unrestricted arm. Eur J Appl Physiol. 2018;118(3):617-627. doi:10.1 007/s00421-018-3806-2
30. Thiebaud RS, Loenneke JP, Fahs CA, et al. The effects of elastic band resistance training combined with blood flow restriction on strength, total bonefree lean body mass and muscle thickness in postmenopausal women. Clin Physiol Funct Imaging. 2013;33(5):344-352. doi:10.1111/cpf.12033

31. Yasuda T, Fujita S, Ogasawara R, Sato Y, Abe T. Effects of low-intensity bench press training with restricted arm muscle blood flow on chest muscle hypertrophy: a pilot study. Clin Physiol Funct Imaging. 2010;30(5):338-343. doi:10.1111/i.1475-097x.2010.00 949.x

32. Sakamaki MG, Bemben M, Abe T. Legs and trunk muscle hypertrophy following walk training with restricted leg muscle blood flow. J Sports Sci Med. 2011;10(2):338-340. https://www.ncbi.nlm.nih.gov/p mc/articles/PMC3761868/.

33. Laurentino GC, Ugrinowitsch C, Roschel H, et al. Strength training with blood flow restriction diminishes myostatin gene expression. Med Sci Sports Exerc. 2011;44(3):406-412. doi:10.1249/mss.0b013e31 $\underline{8233 b 4 b c}$

34. Karabulut M, Sherk VD, Bemben DA, Bemben MG. Inflammation marker, damage marker and anabolic hormone responses to resistance training with vascular restriction in older males. Clin Physiol Funct Imaging. 2013;33(5):393-399. doi:10.1111/cpf.12044

35. Karabulut M, Bemben DA, Sherk VD, Anderson MA, Abe T, Bemben MG. Effects of high-intensity resistance training and low-intensity resistance training with vascular restriction on bone markers in older men. Eur J Appl Physiol. 2011;108(11):1659-1667. doi:10.1007/s00421-010-179 $\underline{6-9}$

36. Neto GR, Novaes JS, Salerno VP, et al. Acute effects of resistance exercises with continuous and intermittent blood flow restriction on hemodynamic measurements and perceived exertion. Percept Mot Skills. 2016;124(1):277-222. doi:10.1177/00315125166 $\underline{77900}$

37. Lixandrão ME, Roschel H, Ugrinowitsch C, Miquelini M, Alvarez IF, Libardi CA. Blood-flow restriction resistance exercise promotes lower pain and ratings of perceived exertion compared with either high- or low intensity resistance exercise performed to muscular failure. J Sport Rehabil. 2019;28(7):706-710. doi:10.1123/jsr.2018-0030

38. Silva JCG, Aniceto RR, Oliota-Ribeiro LS, Neto GR, Leandro LS, Cirilo-Sousa MS. Mood effects of blood flow restriction resistance exercises among basketball players. Percept Mot Skills. 2018;125(4):788-801. doi:1 $\underline{0.1177 / 0031512518776847}$ 
39. da Silva JCG, Silva KF, Domingos-Gomes JR, et al. Aerobic exercise with blood flow restriction affects mood state in a similar fashion to high intensity interval exercise. Physiol Behav. 2019;211:112677. do i:10.1016/j.physbeh.2019.112677

40. Mattocks KT, Mouser JG, Jessee MB, et al. Perceptual changes to progressive resistance training with and without blood flow restriction. J Sports Sci. 2019;37(16):1857-1864. doi:10.1080/02640414.2019.1 $\underline{599315}$

41. Fioriello D, Hillegass E. Interventions and prevention measures for individuals with cardiovascular disease or risk of disease. In: Hillegass E, ed. Essentials of Cardiopulmonary Physical Therapy. 4th ed. St. Louis, Missouri: Elsevier; 2017:568-604.
42. Noordin S, McEwen JA, Kragh CJF Jr, Eisen A, Masri BA. Surgical tourniquets in Orthopaedics. J Bone Joint Surg Am. 2009;91(12):2958-2967. doi:10.21 06/jbjs.i.00634

43. Graham B, Breault MJ, McEwen JA, McGraw RW. Occlusion of arterial flow in the extremities at subsystolic pressures through the use of wide tourniquet cuffs. Clin Orthop Relat Res.

1993;(286):257-261. doi:10.1097/00003086-19930100 $\underline{0-00038}$

44. Okita K, Takada S, Morita N, et al. Resistance training with interval blood flow restriction effectively enhances intramuscular metabolic stress with less ischemic duration and discomfort. Appl Physiol Nutr Metab. 2018;44(7):759-764. doi:10.1139/ apnm-2018-0321 


\section{SUPPLEMENTARY MATERIALS}

\section{Appendix 1}

Download: https://ijspt.scholasticahq.com/article/25791-the-systemic-effects-of-blood-flow-restriction-training-asystematic-review/attachment/65442.docx 